\title{
Effects of auricular therapy to body composition in young asian women
}

\author{
Ching-Hsiu Hsieh ${ }^{1, a}$, Tzu-Jung Tseng ${ }^{2}$, Mei-Yu Huang ${ }^{3}$ and Pei-Ying Chuang ${ }^{4}$ \\ ${ }^{1}$ Department of Nursing, Chang Gung University Of Science and Technology, Assistant Professor, Chiayi, Taiwan \\ ${ }^{2}$ Department of Nursing, Chang Gung University Of Science and Technology, Doctor, Chiayi, Taiwan \\ ${ }^{3}$ Department of Nursing, Yuanpei University of Medical Technology, Hsinchu, Taiwan \\ ${ }^{4}$ School of Nursing, University of Maryland, Assistant Professor, USA
}

\begin{abstract}
This study investigated the efficacy of auricular therapy using semen vaccariae (SV) via auricular point sticking on weight loss, changes to waist circumference, and waist-to-hip ratio.

The study used a randomized design with one control group and one experimental group consisting of young Asian women with waist circumferences $\geq 80 \mathrm{~cm}$.

The total sample size was 50 young women who ranged in age from 18 to 20 years-old.

Each participant attended weekly ten-minute sessions during which auricular acupressure treatment was performed. During treatment, the control group had $0.5 \mathrm{~cm}^{2}$ adhesive tape placed onto their auricular acupoints, while the experimental group had the SV stick applied to the auricular acupoints. Dependent variables were collected at baseline and follow-up for four weeks.

The control group showed significantly increased body weight $(+0.08 \mathrm{~kg}, \mathrm{p} \leq 0.05)$ and waist circumference $(+0.20 \mathrm{~cm}, \mathrm{p} \leq 0.05)$; however, the $\mathrm{SV}$ group showed significantly decreased body weight $(-1.9120 \mathrm{~kg}, \mathrm{p} \leq 0.05)$, waist circumference $(-4.9200 \mathrm{~cm}, \mathrm{p} \leq 0.05)$ and waist-tohip ratio $(-0.0297, \mathrm{p} \leq 0.05)$ during four weeks of treatment.

Auricular acupressure with SV may be an efficacious option to include in weight loss programs for young women.
\end{abstract}

\section{Introduction}

Many studies have shown that excess weight and obesity, especially abdominal obesity, are associated with numerous chronic health problems, increased mortality rates of cardiovascular or cancer, and depressive symptoms or moderate-to-severe depressive symptoms [1-3].

In the United States and worldwide, obesity is a health issue and public health problem [1]. In 2004, the United States Department of Health and Human Services (DHHS) reported that overweight adolescents have a $70 \%$ chance of becoming overweight or obese adults (U.S. DHHS, 2004) [4]. If one or more parents are overweight or obese, the risk of becoming overweight or obese in adulthood increases to $80 \%$ [5]. Thus, to prevent adult obesity, strategies must target young adults [4]. Not only is auricular acupressure of traditional Chinese medicine remedies convenient, safe and effective, this method can be used to treat obesity-related symptoms such as swelling, constipation, insomnia, dysmenorrhea, headache and digestive illness. Due to the relative safety and broad applications of this

\footnotetext{
${ }^{\mathrm{a}}$ Corresponding author : chinghsiuh@yahoo.com
} 
technique, auricular acupressure has become a popular weight loss method; however, the extent of its efficacy for weight loss and obesity treatment has not been fully studied.

Waist circumference (WC) is an anthropometric measure of central obesity in population studies [6] because high WC values are also positively correlated with cardiovascular complications [7]. According to the classifications adopted by the American Heart Association (AHA) and the National Heart, Lung, and Blood Institute (NHLBI) of the United States, elevated WC in males is defined as equal to or greater than $102 \mathrm{~cm}$ and is defined in females as equal to or greater than $88 \mathrm{~cm}$ (AHA and NHLBI, 2009) [8]. For the purpose of this study, WC $\geq 80 \mathrm{~cm}$ in females was used, conforming to the Bureau of Health Promotion, Department of Health, R.O.C. (Taiwan) (2006) [9].

In traditional Chinese Medicine (TCM), "Qi" is thought to be the energy that circulates throughout the body. Traditional acupuncturists activate Qi by inserting needles into specific points (acupoints) on the body [10-12]. Practitioners of Chinese medicine believe that an "excess" or "deficiency" of Qi can be normalized by the specific stimulation of select acupoints [10-13]. Also, acupoint stimulation activates the metabolism of individuals [14]. One of the tenets of TCM is that acupuncture treatment may modify central nervous system neurotransmitter levels through the stimulation of acupoints [1011].

Auricular acupuncture or acupressure therapy has been used as an adjunctive therapeutic modality in complementary medicine practice worldwide [10-16]. In TCM, acupoints of the ear are believed to connect to the internal organs of the human body [17, 23]. Auricular acupuncture stimulation utilizes needles, pellets (Semen Vaccariae), magnetic pearls and other materials. In many countries, auricular acupuncture therapy is the TCM method most frequently used for obesity treatment [18]. A large body of research shows that the most effective auricular acupuncture points for treating obesity are the Shenmen, Mouth, Stomach, Endocrine, and Small Intestine points. Specifically, stimulating the Shenmen point calms patients and induces a degree of sedation. Stimulating the auricular mouth acupoint reduces feelings of anger and has been used to treat oral ulcers. The stomach and endocrine auricular points diminish appetite and reduce the sensation of hunger, which causes feelings of satiety and fullness, promoting weight loss [1,17-23]. The small intestine point treats dyspepsia, which may ultimately contribute to weight loss [23].

The plant pellet Semen Vaccariae (SV), termed Wang-Bu-Liu-Xing by the Chinese and commonly known as vaccaria seeds, are the dried seeds of Vaccaria segetalis ripe fruit. More than thousands of years ago, Chinese medicine was used to treat amenorrhoea and breast infections [23]. One study reported the systematic analysis of SV metabolism and the distribution of its chemical constituents after oral administration [24]. Also, SV is comprised of small round seeds that are commonly used in auricular taping, and the average SV size is approximately $0.13 \mathrm{~cm}$ [23]. SV is easily purchased from every Chinese herb market. SV sticks apply pressure to the skin both via acupuncture and finger pressure treatment when placed onto the skin points. In general, auricular therapy using SV has been found to be cost-effective and hygienic, with few side effects; therefore, SV may be more appealing to clients than many other complementary therapies for weight reduction [23].

\section{Methods}

\subsection{Demographics and study design}

This study was designed to test the effects of auricular acupressure by SV on body composition factors such as weight loss, WC and waist-to-hip ratio (WHR) in young Asian females. The total intervention duration was four weeks. The sample consisted of 55 participants who ranged in age from 18 to 20 years-old and were recruited from universities in northern Taiwan. The participants were randomly assigned to one of two groups (control or experimental) by a computer-generated list, which was held confidential; therefore, no one except the principal investigator knew the treatment allocation of the patients. Ethical approval was obtained from their schools, and informed consent was obtained from each participant. All participants were free to withdraw from the study at any time. Potential 
benefits of the study included a reduction in participant weight and $\mathrm{WC}$, which have the potential to improve health status and overall well-being.

\subsection{Intervention}

For the purpose of this study, a WC $\geq 80 \mathrm{~cm}$ in females was used as a selection criterion because this parameter conforms with the Bureau of Health Promotion, Department of Health, R.O.C. (Taiwan) (2006) criteria. The participants were randomly assigned to one of two groups. Both groups received health education describing an appropriate reduced calorie diet and lifestyle modifications such as methods to increase activity levels. During treatment, the control group had adhesive tape $\left(\mathrm{a} 0.5 \mathrm{~cm}^{2}\right.$ piece of tape) placed onto their auricular acupoints, while the experimental group received the SV stick applied onto the auricular acupoints. The auricular acupoints used were the Shenmen, Mouth, Stomach, Small Intestine and Endocrine points. Each week, the SV or adhesive tape was placed onto one ear, and the following week, the SV or tape only was applied to the other ear. No specific changes to diet or physical activity were prescribed. Each participant met with the study coordinators once a week for ten minutes for a total of four weeks. Body composition measurements, including body weight, WC, hip circumference and WHR, were recorded for each participant each week as shown in Figure 1, which depicts a flow chart of the study design.

\section{Data Analysis}

The data were analyzed using the SPSS software (SPSS 19.0. for Windows) the General Linear Mixed-Effects Model (GLMM). Weight loss, WC and WHR measurements were collected for each group at each weekly meeting and were also assessed for significance. Mean and standard deviations of BW, WC and WHR score were compared among subjects who were treated with each group. In the primary study outcome measures of differences between both groups were analyzed using linear mixed model analysis of variance with group and meeting time as fixed effects. for continuous outcomes. Normally distributed variables and generalized estimating equations for categorical variables. The results were considered to be significant at $p<0.05$.

\section{Results}

Table 1. Mean difference in body weight $(\mathrm{kg})$ from baseline to the completion of the intervention $(\mathrm{N}=50)$.

\begin{tabular}{c|c|c|c|c|}
\hline Week & Control Group & $\mathrm{p}$ & Experimental Group & $\mathrm{p}$ \\
\hline & $\begin{array}{c}\text { Body Weight Mean } \\
\text { Difference }\end{array}$ & $\begin{array}{c}\text { Body Weight Mean } \\
\text { Difference }\end{array}$ & \\
\hline Baseline - 1st Week & +0.1080 & $* \leq 0.05$ & -0.4680 & $* \leq 0.05$ \\
\hline Baseline - 2nd Week & +0.0680 & $* \leq 0.05$ & -1.1440 & $* \leq 0.05$ \\
\hline Baseline - 3rd Week & +0.1120 & $* \leq 0.05$ & -1.6240 & $* \leq 0.05$ \\
\hline Baseline - 4th Week & +0.0800 & $* \leq 0.05$ & -1.9120 & $* \leq 0.05$ \\
\hline
\end{tabular}

$* \mathrm{p} \leq 0.05$

After four weeks of auricular therapy, 5 participants had withdrawn from the study for a variety of reasons, and these 5 subjects were not included in the final analysis. The final analysis includes 25 subjects in the control group and 25 subjects in the treatment group.

In the control group, the average body weight remained the same during the first two weeks $(\mathrm{BW}=+0.0680 \mathrm{~kg})$; however, the average body weight significantly increased $(\mathrm{BW}=+0.0800 \mathrm{~kg})$ during the third and fourth observation weeks $(\mathrm{p} \leq 0.05)$. In contrast, in the experimental group, the average body weight was significantly reduced $(\mathrm{p} \leq 0.05)$ from the first week to the fourth week (BW $=-0.4680 \mathrm{~kg}$ during the first week; $-1.1440 \mathrm{~kg}$ during the second week; $-1.6240 \mathrm{~kg}$ during the third 
week; and $-1.9120 \mathrm{~kg}$ during the fourth week) as shown in Table 1. Furthermore, the participants who received auricular acupressure treatment with the SV were noted to have decreased body weight from week-to-week.

The WC was also measured weekly during the four-week program duration. After four weeks, the WC of the control group increased significantly by $+0.2000 \mathrm{~cm}(\mathrm{p} \leq 0.05)$; therefore, similar to the overall body weight, simply applying adhesive tape to auricular acupoints increased the WC after four weeks (Table 2). In contrast, the WC of the treatment group decreased significantly by $-4.9200 \mathrm{~cm}(\mathrm{p}$ $\leq 0.05$ ), and the participants who received auricular acupressure with SV were noted to have decreased WC from week-to-week.

Table 2. Mean difference of WC $(\mathrm{cm})$ from baseline to the completion of the intervention $(\mathrm{N}=50)$

\begin{tabular}{c|c|c|c|c|}
\hline Week & Control Group & $\mathrm{p}$ & Experimental Group & $\mathrm{p}$ \\
\hline & $\begin{array}{c}\text { WC } \\
\text { Mean Difference }\end{array}$ & & $\begin{array}{c}\text { WC } \\
\text { Mean Difference }\end{array}$ & \\
\hline Baseline $-1^{\text {st }}$ Week & +0.4400 & .000 & -1.0480 & $* \leq 0.05$ \\
\hline Baseline $-2^{\text {nd }}$ Week & +0.3800 & .000 & -2.6000 & $* \leq 0.05$ \\
\hline Baseline $-3^{\text {rd }}$ Week & +0.7000 & .000 & -3.6800 & $* \leq 0.05$ \\
\hline Baseline $-4^{\text {th }}$ Week & +0.2000 & .000 & -4.6800 & $* \leq 0.05$ \\
\hline
\end{tabular}

$* \mathrm{p} \leq 0.05$

The WHR was also measured weekly. After four weeks, the WHR of the control group showed a non-significant increase by $0.0003(p=0.728)$, which is in contrast to the body weight and WC, which increased significantly in the control group (Table 3). The WHR of the SV treatment group decreased significantly by 0.0297 ( $\mathrm{p} \leq 0.05$ ) over the course of the study. The participants who received continuous auricular acupressure treatment with SV were noted to have decreased WHR from weekto-week (Table 3).

Table 3. Mean difference of WHR from baseline to the completion of the intervention $(\mathrm{N}=50)$

\begin{tabular}{c|c|c|c|c|}
\hline Week & Control Group & $\mathrm{p}$ & Experimental Group & $\mathrm{p}$ \\
\hline & $\begin{array}{c}\text { WHR } \\
\text { Mean Difference }\end{array}$ & & $\begin{array}{c}\text { WHR } \\
\text { Mean Difference }\end{array}$ & \\
\hline Baseline $-1^{\text {st }}$ Week & +0.0009 & .496 & -0.0070 & $* \leq 0.05$ \\
\hline Baseline $-2^{\text {nd }}$ Week & -0.0007 & .782 & -0.0172 & $* \leq 0.05$ \\
\hline Baseline $-3^{\text {rd }}$ Week & +0.0038 & .951 & -0.0199 & $* \leq 0.05$ \\
\hline Baseline $-4^{\text {th }}$ Week & +0.0003 & .728 & -0.0297 & $* \leq 0.05$ \\
\hline
\end{tabular}

$* \mathrm{p} \leq 0.05$

\section{Discussion}

The current study demonstrates that after 4 weeks of auricular acupressure treatment using SV, the body weight of the participants significantly decreased. This result confirmed that repeated auricular acupressure decreases body weight, which has been supported by other research studies. Chien and colleagues (2011) used electrical stimulation of acupoints on 21 obese patients over treatment periods ranging from 12 weeks and reported weight loss ranging from $63.48 \pm 4.9 \mathrm{~kg}$ to $62.40 \pm 4.81 \mathrm{~kg}$, decreased waist circumference from $84.25 \pm 2.69 \mathrm{~cm}$ to $82.33 \pm 2.27 \mathrm{~cm}$, and decreased waist to hip ratio from $0.84 \pm 0.03$ to $0.83 \pm 0.02$ [17]. Additionally, Richards and Marley (1998) assessed the efficacy of ear acupuncture treatment on weight loss in a 4-week study, and their results showed an average weight loss of $4.0 \pm 1.4 \mathrm{~kg}(p<0.05)$ in the treatment group [20]. 
Beyond its effects on weight loss, this study suggests that acupressure may have additional benefits to anthropometry. Lee, Jacobs, Schreiner, Iribarren and Hankinson (2007) showed that WC and WHR predict coronary artery calcification (CAC). In the current study, 4 weeks of auricular acupressure treatment using SV significantly decreased participant WC and WHR, which may then translate to reduced risk of CAC [24].

\section{Conclusion}

Cho and colleagues (2009) reported that stimulation via acupuncture, acupressure and heating (moxibustion) can be used to treat obesity [26]. Reduction of body weight and decreasing the prevalence of obesity in society are critical health issues [25]. Research has shown that a relationship exists between abdominal obesity and subclinical atherosclerotic vascular disease in multiple race, sex and age groups. Abdominal obesity, measured by waist size or WHR, is related with early atherosclerosis as measured by the presence of CAC [24]. Thus, the current study is important because the findings show that abdominal obesity can be safely and effectively reduced by auricular acupressure.

Other auricular acupoints will be considered in the future study. The sample size of our study is similar to the size in many previous research studies that reached similar conclusions. However, the participants in the current study were not representative of both genders. This study was exclusively comprised of women participants, and future studies should specifically recruit male participants to better reflect the general population.

In summary, auricular acupressure using SV decreased body weight, WC and WHR after just four weeks of treatment. Therefore, auricular acupuncture offers an effective and economical alternative for the treatment of obesity in contrast to more conventional treatment methods such as exercise, diet control, medicine and surgery. However, the effects of long-term treatment and follow-up after treatment are necessary for future studies. Future studies should also consider the level of baseline body weight on the effectiveness of the treatment, and the extent to which body weight can be modulated by auricular acupressure with SV.

\section{References}

1. S. K. Kumanyika, E. Obarzanek, N. Stettler, R. Bell, A. E. Field, S. P. Fortmann, B. A. Franklin, M. W. Gillman, C. E. Lewis, W. C. Poston, J. Stevens, and Y. Hong, Population-based prevention of obesity: the need for comprehensive promotion of healthful eating, physical activity, and energy balance: a scientific statement from American Heart Association Council on Epidemiology and Prevention, Interdiscuplinary Committee for Prevention, Circulation 118 (4), 428-464 (2008)

2. G. Zhao, E. S. Ford, C. Y. Li, J. Tsai, S. Dhingra, and L. Balluz, Waist circumference, abdominal obesity, and depression among overweight and obese U.S. adults: national health and nutrition examination survey 2005-2006, BMC Psychiatry 11, 130 (2011)

3. C. Zhang, K. M. Rexrode, R. M. VanDam, T. Y. Li, and F. B. Hu, Abdominal obesity and th risk of all-cause, cardiovascular, and cancar mortality: sixteen years of follow-up in US women, Circulation, 117 (13), 1658-1667 (2008)

4. National Center for Chronic Disease Prevention and Health Promotion, Defining overweight and obesity, http://www.cdc.gov/nccdphp/dnpa/obesity/defining.htm (2004)

5. National Health and Nutrition Examination Survey (NHANES) 1999-2000, Hyattsville, MD, US Department of Health and Human Services, Centers for Disease Control and Prevention, National Center for Health Statistics (2002)

6. R. O. Moreira, K. F. Marca, J. C. Appolinario, and W. F. Coutinho, Increased waist circumference is associated with an increased prevalence of moon disorders and depressive symptoms in obese women, Eating and weight Disorders, 12 (1), 35-40 (2007) 
7. A. Onat, G. S. Avei, M. M. Barlan, H. Uyarel, B. Uzunlar, and V. Sanaoy, Measures of abdominal obesity assessed for visceral adiposity and relation to coronary risk, International Journal of Obesity and Related Metabolic Disorders, 28 (8), 1018-1025 (2004)

8. American Heart Association and the National Heart, Lung and Blood Institute, Metabolic Syndrome, http://www.americanheart.org/presenter.jhtml?identifier=4756 (2009)

9. Bureau of Health Promotion, Department of Health, R.O.C.(Taiwan), Metabolic Syndrome, http://www.bhp.doh.gov.tw/BHPnet/Portal/Them_Show.aspx?Subject=200712250023\&Class=2 $\&$ No=200712250120 (2006)

10. T. Oleson, Differential application of auricular acupuncture for myofascial, autonomic, and naturopathic pain, Medical Acupuncture, 9, 23-28 (1998).

11. T. Oleson, Auriculotherapy stimulation for neuro-rehabilitation, NeuroRehabilitation, 17 (1), 49$62(2002)$

12. A. Vickers and C. Zollman, ABC of complementary medicine: acupuncture, British Med. J., 319 (7215), 973-976 (1999)

13. E. R. Mitchell, Fighting drug abuse with acupuncture: the treatment that works, Berkeley: Pacific View Press (1995)

14. J. Li, Clinical experience in acupuncture treatment of obesity, Journal of Traditional Chinese Medicine, 19 (1), 48-51 (1999)

15. B. L. Frank and N. Soliman, Obesity treatment through auricular therapy and auricular medicine, Medical Acupuncture, 14 (1), 33-35 (2002)

16. C. H. Hsieh, The effects of auricular acupressure on weight loss and serum lipid levels in overweight adolescents, The American Journal of Chinese Medicine, 38 (4), 675-682 (2010)

17. L. W. Chien, M. H. Lin, H. Y. Chung, and C. F. Liu, Transcutanous electrical stimulation of acupoints changes body composition and heart rate variability in postmenopausal women with obesity, Evidence-Based Complementary and Alternative Medicine, 2011 (9), 862121 (2011)

18. G. Stux and B. Pomeranz, Basics of acupuncture, 4th ed. Springer-Verlag: Berlin (1998)

19. R. Mazzoni, E. Mannucci, S. M. Rizzllo, V. Ricca, and C. M. Rotella, Failure of acupuncture in the treatment of obesity: a pilot study, Eating Weight Disorders, 4 (4), 198-202 (1999)

20. D. Richards and J. Marley, Stimulation of auricular acupuncture points in weight loss, Australia Family Physician, 27 (2), S73-S77 (1998)

21. T. S. Shafshak, Electroacupuncture and exercise in body weight reduction and their application in rehabilitating patients with knee osteoarthritis, The American Journal of Chinese Medicine, 23 (1), 15-25 (1995)

22. R. P. Steiner, N. Kupper, and A. W. Davis, Obesity and appetite control: comparison of acupuncture therapies and behavior modification, Proc. of International Forum on Family Medicine Education, Society of Teachers of Family Medicine, Kansas City, 313-326 (1983)

23. S. N. Chang, Chinese Acupuncture, 3rd ed. Beijing: People Sanitation (1995)

24. S. Sang, A. Lao, Z. Chen, J. Uzawa, and Y. Fujimoto, Chemistry and bioactivity of the seeds of Vaccaria segetalis, in: Oriental Foods and Herbs, American Chemical Society, 279-291 (2003)

25. C. D. Lee, D. R. Jacobs Jr., P. J. Schreiner, C. Iribarren, and A. Hankinson, Abdominal obesity and coronary artery calcification in young adults: the coronary artery risk development in young adults (CARDIA) study, The American Journal of Clinical Nutrition, 86 (1), 48-54 (2007)

26. J. M. Ripp and S. Hess, The role of physical activity in the prevention and management of obesity, Journal of the American Dietetic Association, 98 (10), S31-S38 (1998)

27. S. H. Cho, J. S. Lee, L. Thabane, and J. Lee, Acupuncture for obesity: a systematic review and meta-analysis, International Journal of Obesity, 33 (2), 183-196 (2009) 